\title{
SBRT for the primary treatment of localized prostate cancer: the effect of Gleason score, dose and heterogeneity of intermediate risk on outcome utilizing 2.2014 NCCN risk stratification guidelines
}

\section{Matthew Bernetich 1,2, Caspian Oliai ${ }^{2}$, Rachelle Lanciano ${ }^{2,3} *$, Alexandra Hanlon ${ }^{4}$, John Lamond ${ }^{2,3}$, Stephen Arrigo ${ }^{2,3}$, Jun Yang ${ }^{2,3}$, Michael Good ${ }^{2}$, Jing Feng ${ }^{2}$, Royce Brown ${ }^{2}$, Bruce Garber ${ }^{2}$, Michael Mooreville ${ }^{2}$ and Luther W. Brady ${ }^{2,3}$}

${ }^{1}$ Rowan University School of Osteopathic Medicine, Stratford, NJ, USA

2 Philadelphia CyberKnife Center, Delaware County Memorial Hospital, Havertown, PA, USA

${ }^{3}$ Department of Radiation Oncology, Drexel University College of Medicine, Philadelphia, PA, USA

${ }^{4}$ Office of Nursing Research, School of Nursing, University of Pennsylvania, Philadelphia, PA, USA

\section{Edited by:}

Alan Jay Katz, Flushing Radiation

Oncology, USA

\section{Reviewed by:}

Kara Leonard, Rhode Island Hospital, USA

Matthew Charles Abramowitz,

University of Miami, USA

*Correspondence:

Rachelle Lanciano, Philadelphia CyberKnife Center, 2010 West

Chester Pike Suite 115, Havertown, PA 19083, USA

e-mail: rlancmd@gmail.com
Purpose: To report an update of our previous experience using stereotactic body radiation therapy (SBRT) for the primary treatment of prostate cancer, risk stratified by the updated National Comprehensive Cancer Network (NCCN) version 2.2014, reporting efficacy and toxicity in a community hospital setting.

Methods: From 2007 to 2012, 142 localized prostate cancer patients were treated with SBRT using CyberKnife. NCCN guidelines Version 2.2014 risk groups analyzed included very low $(20 \%)$, low $(23 \%)$, intermediate $(35 \%)$, and high (22\%) risk. To further explore group heterogeneity and to comply with new guidelines, we separated our prior intermediate risk group into favorable intermediate and unfavorable intermediate groups depending on how many intermediate risk factors were present (one vs. > one). The unfavorable intermediate group was further analyzed in combination with the high risk group as per NCCN guidelines Version 2.2014. Various dose levels were used over the years of treatment, and have been categorized into low dose ( $35 \mathrm{~Gy}, n=5$ or $36.25 \mathrm{~Gy}, n=107$ ) and high dose (37.5 Gy, $n=30$ ). All treatments were delivered in five fractions. Toxicity was assessed using radiation therapy oncology group criteria.

Results: Five-year actuarial freedom from biochemical failure (FFBF) was 100, 91.7, 95.2, 90.0, and $86.7 \%$ for very low, low, intermediate and high risk patients, respectively. A significant difference in 5 year FFBF was noted for patients with Gleason score (GS) $\geq 8$ vs. 7 vs. $5 / 6(p=0.03)$ and low vs. high dose $(p=0.05)$. T-stage, pretreatment PSA, age, risk stratification group, and use of ADT did not affect 5-year FFBF. Multivariate analysis revealed GS and dose to be the most predictive factors for 5-year FFBF.

Conclusion: Our experience with SBRT for the primary treatment of localized prostate cancer demonstrates favorable efficacy and toxicity comparable to the results reported for IMRT in literature. GS remains the single most important pretreatment predictor of outcome.

Keywords: stereotactic body radiation therapy, prostate cancer, CyberKnife, hypofractionation, NCCN guidelines, risk stratification

\section{INTRODUCTION}

The American Society of Radiation Oncology (ASTRO) model policy update of 2013 (1) acknowledged that stereotactic body radiation therapy (SBRT) is equivalent to standard radiation modalities used to treat prostate cancer such as intensity modulated radiation therapy (IMRT). In addition, the National Comprehensive Cancer Network (NCCN) (2) "cautiously considers SBRT as an alternative radiation treatment strategy to conventional fractionated regimens at clinics with appropriate technology, physics and clinical expertise" (p.707). For low and intermediate risk prostate cancer, recent published literature (3-6) supports these recommendations with excellent 5 year biochemical control rates and correspondingly acceptable rates of toxicity.

Prognostic factors necessary to guide staging and treatment options for prostate cancer include Gleason score (GS), initial PSA, and T-stage. These factors have been used to group patients into risk categories of low, intermediate, and high risk, which 
correlate with outcome in radical prostatectomy and definitive radiation series $(7,8)$. More recently, clinical criteria have been introduced to predict pathologically "insignificant" prostate cancer, which is defined as clinical stage T1c, GS $\leq 6$, disease in fewer than 3 biopsy cores, and less than 50\% involvement in any core with PSA density $<0.15 \mathrm{ng} / \mathrm{mL} / \mathrm{g}(9-11)$. In addition, the Memorial Sloane-Kettering group identified high-risk features within the intermediate risk group, which correlated with decreased biochemical freedom from relapse, decreased local control, increased distant metastasis, and increased prostate-specific mortality. They created stratifications of favorable and unfavorable intermediate risk groups, assigning the unfavorable intermediate risk group to patients with primary Gleason pattern of 4, percentage of biopsy positive cores $\geq 50 \%$, or multiple NCCN-defined intermediate risk factors (cT2b-c, PSA 10-20, or GS 7) (12).

The NCCN Prostate Cancer Panel recognized that heterogeneity exists within the previous risk stratification groups and has redesigned guidelines for prostate cancer by risk categories, which are linked to treatment paradigms with the hope that these updated risk groups can be used more accurately to begin discussing treatment options with prostate cancer patients. These updated NCCN guidelines, version 2.2014, stratify prostate cancer into five risk groups (very low, low, intermediate, high, and very high) utilizing GS, initial PSA, and T-stage $(2,13)$. These guidelines separate out "insignificant" very low risk prostate cancer and suggest raising the risk group depending on number of risk factors within each group. Clinical stage T2c is now considered intermediate risk by NCCN (2) compared to the D'Amico classification, which places T2c patients into the high risk category $(7,8)$.

We report an update of our previously published experience with CyberKnife SBRT (14), assessing efficacy and toxicity while exploring the new NCCN risk groups in a consecutively treated database of early stage prostate cancer. We have adopt the Memorial group's favorable and unfavorable intermediate risk stratification but simplify the unfavorable intermediate group to patients who demonstrate multiple NCCN-defined intermediate risk factors to conform to the new NCCN guidelines.

\section{MATERIALS AND METHODS}

One hundred forty-two early stage prostate cancer patients received SBRT at the Philadelphia CyberKnife Center from 2007 through 2012. The median and mean follow-up were 38 and 39 months, respectively, with a range of $0-76$ months. This IRBapproved retrospective study included updated information on 70 patients from our previous experience, in addition to 72 patients treated thereafter. SBRT was delivered using the CyberKnife (Accuray Inc., Sunnyvale, CA, USA) with MultiPlan inverse treatment planning and motion tracking of internal fiducials. Treatment planning began with transrectal or transperineal ultrasoundguided placement of four gold fiducials into the prostate. A CT scan (1.25-mm slice thickness) was obtained 10-14 days later to allow inflammation to subside and to ensure fiducials did not migrate. T2 weighted turbo spin-echo MRI was obtained for contouring the prostate and seminal vesicles after registration by fiducials with CT scan and T1 vibe fat suppression MRI.

The prostate, seminal vesicles, rectum, bladder, penile bulb, testes, and sigmoid colon were contoured (Figure 1). Urethra

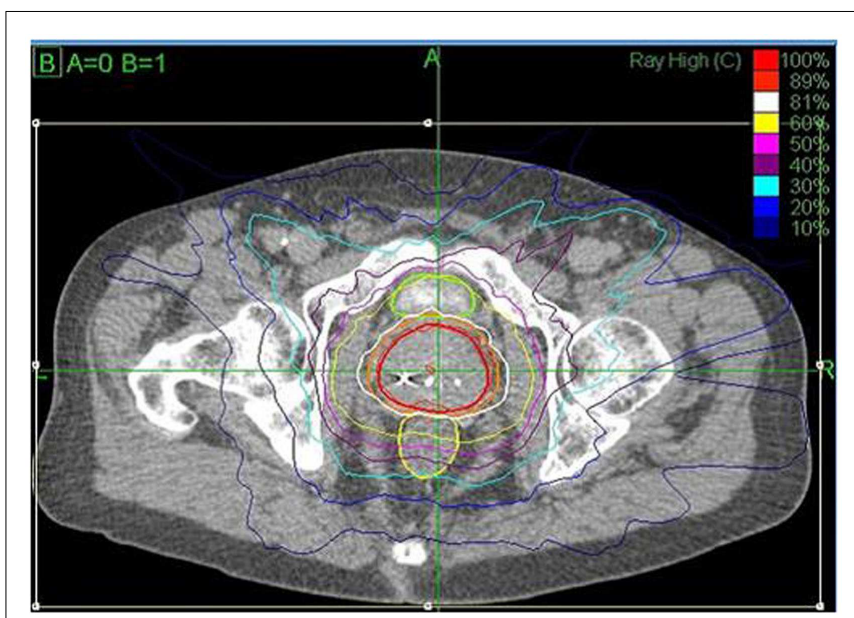

FIGURE 1 | CyberKnife SBRT treatment plan (36.25 Gy $\times 5$ fractions prescribed to the $81 \%$ isodose line (white) with a $\mathbf{4 4 . 7 5} \mathbf{G y}$ maximum dose). Shown are the prostate $\left(43.6 \mathrm{~cm}^{3}\right.$, red) and PTV $\left(77.8 \mathrm{~cm}^{3}\right.$, orange). A bladder (green) volume of $1.66 \mathrm{~cm}^{3}$ received $37 \mathrm{~Gy}$ and $0.4 \mathrm{~cm}^{3}$ of the rectum (yellow) received $36 \mathrm{~Gy}$.

contouring was not required. The clinical target volume (CTV) was the prostate for low risk patients and the prostate plus $2-\mathrm{cm}$ seminal vesicle base for intermediate/high risk patients. A total dose of $35,36.25$, or $37.5 \mathrm{~Gy}$, delivered in five fractions, was prescribed to the planning target volume (PTV) that consisted of the CTV with a $5 \mathrm{~mm}$ margin in all directions except $3 \mathrm{~mm}$ posteriorly. Patients were typically treated over 8 days with a weekend off in between with an every other day treatment schedule. If necessary because of patient convenience or staff coverage, treatments were consecutive. Our first cohort of patients was treated with $35 \mathrm{~Gy}$ $(n=5)$ with subsequent dose escalation to $37.5 \mathrm{~Gy}(n=30)$ per department protocol and review of radiobiology data available at the time. Most recent cohort was treated with a lower dose of $36.25 \mathrm{~Gy}(n=107)$ after more published experience was available, as well as participation in a national protocol, which required that dose. The dose was not changed due to detected poor outcome or toxicity. The dosimetric goal was to cover at least $95 \%$ of the PTV with the prescribed dose normalized to the $75-85 \%$ isodose line (dose heterogeneity 17-33\%). Less than $1 \mathrm{~cm}^{3}$ of rectum received $36 \mathrm{~Gy}, 50 \%$ of the prescribed dose could not cross the posterior rectal wall, and $<10 \mathrm{~cm}^{3}$ of bladder received $37 \mathrm{~Gy}$. Less than $50 \%$ of the penile bulb received $29.5 \mathrm{~Gy}$ and less than $1 \mathrm{cc}$ of the sigmoid colon received 30 Gy. The average CTV and PTV were 56.9 $\left(\mathrm{SD} 26.1 \mathrm{~cm}^{3}\right.$ ) and $98.3 \mathrm{~cm}^{3}\left(\mathrm{SD} 38.6 \mathrm{~cm}^{3}\right)$, respectively.

Orthogonal $120-\mathrm{kV}$ X-ray image pairs were obtained throughout treatment for use in motion tracking. The real-time prostate position was locked-on by the relative fiducial position on the $\mathrm{X}$ rays. For those patients with evenly distributed fiducials in the prostate quadrants, the prostate's rotation was also tracked and corrections were made in real time.

PSA nadir was defined as the lowest PSA value following SBRT. Biochemical failure (BF) was assessed using the nadir +2 (Phoenix) definition. Toxicity was assessed using the radiation therapy oncology group (RTOG) criteria; acute toxicity 
occurred within 3 months and late toxicity $>3$ months following treatment (15).

Pretreatment factors were assessed for each patient, which included standard risk factors of initial PSA, GS, and clinical stage. Patients were stratified into four risk groups as per NCCN 2.2014 since we had only two very high risk patients who were included with the high risk group. This stratification includes the NCCN "very low" risk group designation, which separates our earlier low risk group into two groups (very low and low). To explore group heterogeneity, we separated our previous intermediate risk group into favorable intermediate if there was one intermediate risk factor (Gleason 7 or clinical stage T2b/c or PSA > 10) or unfavorable intermediate if there was more than one intermediate risk factor. For the purpose of further analysis, the unfavorable intermediate group was then combined with the high risk group as per NCCN version 2.2014.

Kaplan-Meier estimates of freedom from biochemical failure (FFBF) were used to describe the patients overall, and comparisons were accomplished using log-rank statistics $(16,17)$. Simple Cox proportional hazards regression modeling was used to estimate hazard ratios for categorical variables of interest. The overall sample was described using measures of central tendency (mean and median) and variation (standard deviation, IQR). Descriptive statistics were used to describe toxicity within each of the treatment groups.

\section{RESULTS}

\section{PATIENT AND TREATMENT CHARACTERISTICS}

One hundred and forty-two consecutive patients were analyzed with pretreatment and treatment factors described in Table $\mathbf{1}$. NCCN risk groups are depicted in Table 1 with either three (D'Amico classification) or five strata (NCCN version 2.2014 with intermediate risk group stratification and no high risk stratification). Androgen deprivation therapy (ADT) in the form of luteinizing hormone-releasing hormone agonist was used in $28.2 \%$ of patients $(11.5 \%$ of the low risk, $37.7 \%$ of the intermediate risk and $50 \%$ of the high risk). The use of ADT was dependent on individual urologist and radiation oncologist preference. Selected very low and low risk patients received ADT to shrink the prostate before SBRT.

Patients who received 35 or 36.25 Gy were termed the "low-dose group" and those who received 37.5 Gy the "high-dose group." Most patients (57\%) received their treatment over 5-8 days. The remainder completed treatment between 9 and 19 days with one non-compliant patient receiving his final fraction several weeks later, all due to poor adherence to their schedule. Treatment was delivered with an average of $187(\mathrm{SD}=26)$ non-coplanar beams. $\mathrm{X}$-ray images were taken every three to five beams to track the prostate's movement.

\section{PSA RESPONSE AND BIOCHEMICAL CONTROL}

The overall 5-year actuarial FFBF for the whole group was $92.7 \%$ (Table 2). Six patients out of the entire cohort of 142 patients, all in the low dose group, experienced BF; two were high risk, three intermediate risk, and one low risk. The low-dose and high-dose groups' median PSA nadirs were 0.3 and $0.1 \mathrm{ng} / \mathrm{mL}$, respectively. Five year actuarial FFBF was $100 \%$ for the high dose group and
Table 1 | Patient and tumor characteristics

\begin{tabular}{|c|c|c|}
\hline Age at diagnosis & Years & \\
\hline Median (IQR) & $67(61,73)$ & \\
\hline Range & $44-88$ & \\
\hline Pretreatment PSA & $\mathrm{ng} / \mathrm{mL}$ & \\
\hline Mean (SD) & $8.1(7.7)$ & \\
\hline Median (IQR) & $5.7(4.4,8.3)$ & \\
\hline Pretreatment PSA & Number of patients & Percent \\
\hline$<10 \mathrm{ng} / \mathrm{mL}$ & 115 & 81 \\
\hline $10-20 \mathrm{ng} / \mathrm{mL}$ & 19 & 13 \\
\hline$>20 \mathrm{ng} / \mathrm{mL}$ & 8 & 6 \\
\hline T-stage & Number of patients & Percent \\
\hline T1c & 106 & 75 \\
\hline T2a & 19 & 13 \\
\hline$>\mathrm{T} 2 \mathrm{a}$ & 17 & 12 \\
\hline Gleason score & Number of patients & Percent \\
\hline $5-6$ & 76 & 53 \\
\hline 7 & 54 & 38 \\
\hline $8+$ & 12 & 9 \\
\hline Risk Group (3) & Number of patients & Percent \\
\hline Low & 61 & 43 \\
\hline Intermediate & 63 & 44 \\
\hline High & 18 & 13 \\
\hline Risk group (5) & Number of patients & Percent \\
\hline Very low & 28 & 20 \\
\hline Low & 33 & 23 \\
\hline Favorable intermediate & 50 & 35 \\
\hline Unfavorable intermediate & 13 & 9 \\
\hline High & 18 & 13 \\
\hline Dose group & Number of patients & Percent \\
\hline Low & 112 & 79 \\
\hline High & 30 & 21 \\
\hline Hormone treatment & Number of patients & Percent \\
\hline No ADT & 102 & 72 \\
\hline ADT & 40 & 28 \\
\hline
\end{tabular}

$93.75 \%$ for the low dose group, $p=0.05$, hazard ratio of 11.0 for low compared to high dose (Table 2, Figure 2).

We analyzed the heterogeneity of intermediate risk prostate cancer by stratifying into an intermediate favorable and unfavorable as described in the methods with a 5.2\% decrement in 5-year actuarial FFBF from 95.2 to $90.0 \%$. We then compared the intermediate unfavorable with high risk and found a $6.1 \%$ decrement in 5-year actuarial FFBF from 90 to $83.9 \%$. We then grouped the intermediate unfavorable with the high risk group as per version 2.2014 NCCN guidelines with 5-year actuarial FFBF of $100 \%$ for very low risk, $91.7 \%$ for low risk, $95.2 \%$ for intermediate risk, and $86.7 \%$ for high risk (Table 2). Previous NCCN risk categories of low, intermediate, and high 5-year actuarial FFBF are presented also in order to compare to other contemporary series of prostate cancer SBRT (Table 2). Since there was little difference in outcome in our data base between very low, low, and intermediate NCCN version 2.2014 risk categories, they were grouped and compared to high risk with 5 year actuarial FFBF of $94.4 \%$ for very low/low/intermediate and $86.7 \%$ for high $(p=0.07$, Table 2$)$. 
Table 2 | Freedom from biochemical failure stratified by risk and descriptive statistics.

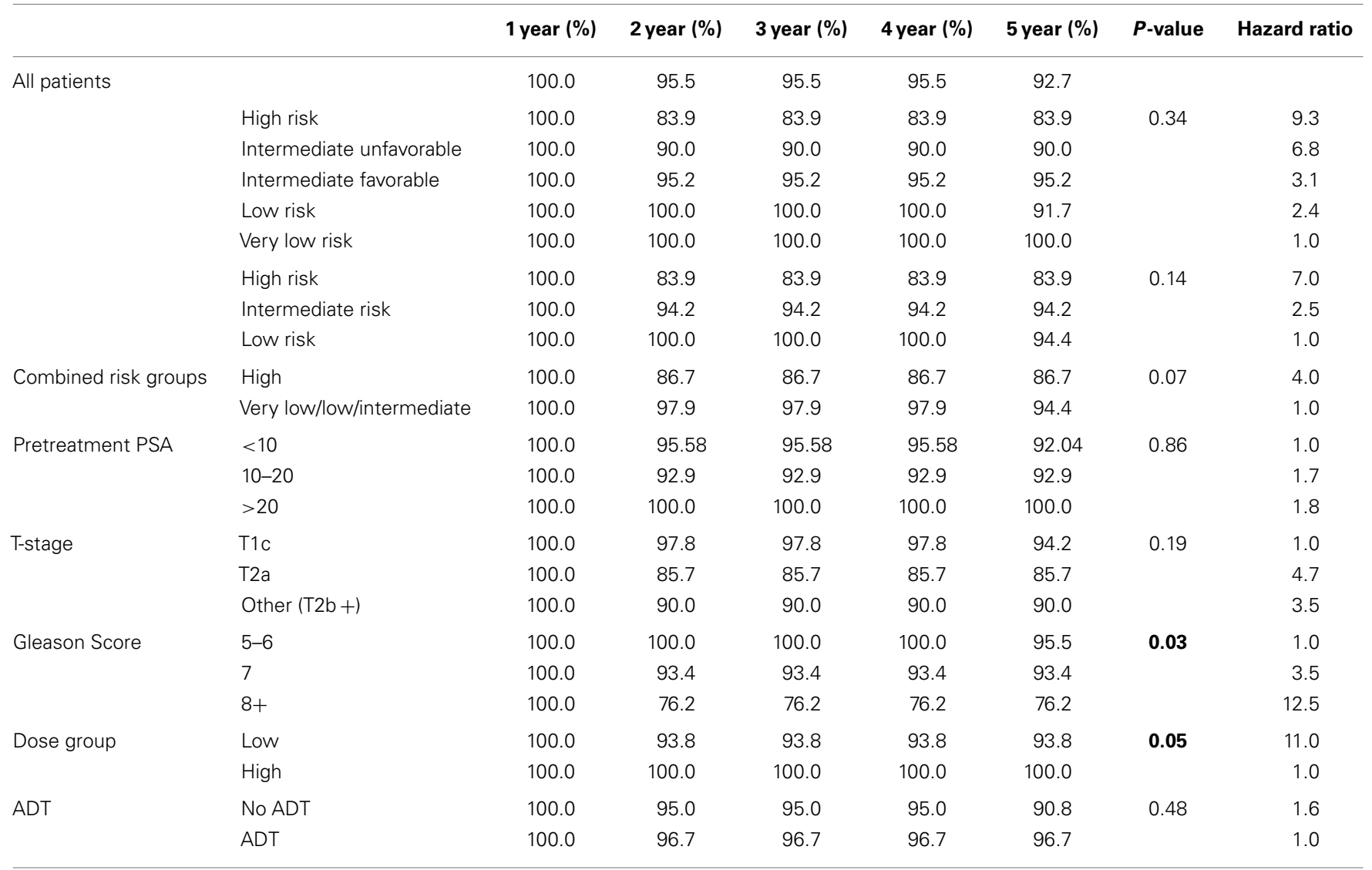

Bold font indicates significant $p$-value.

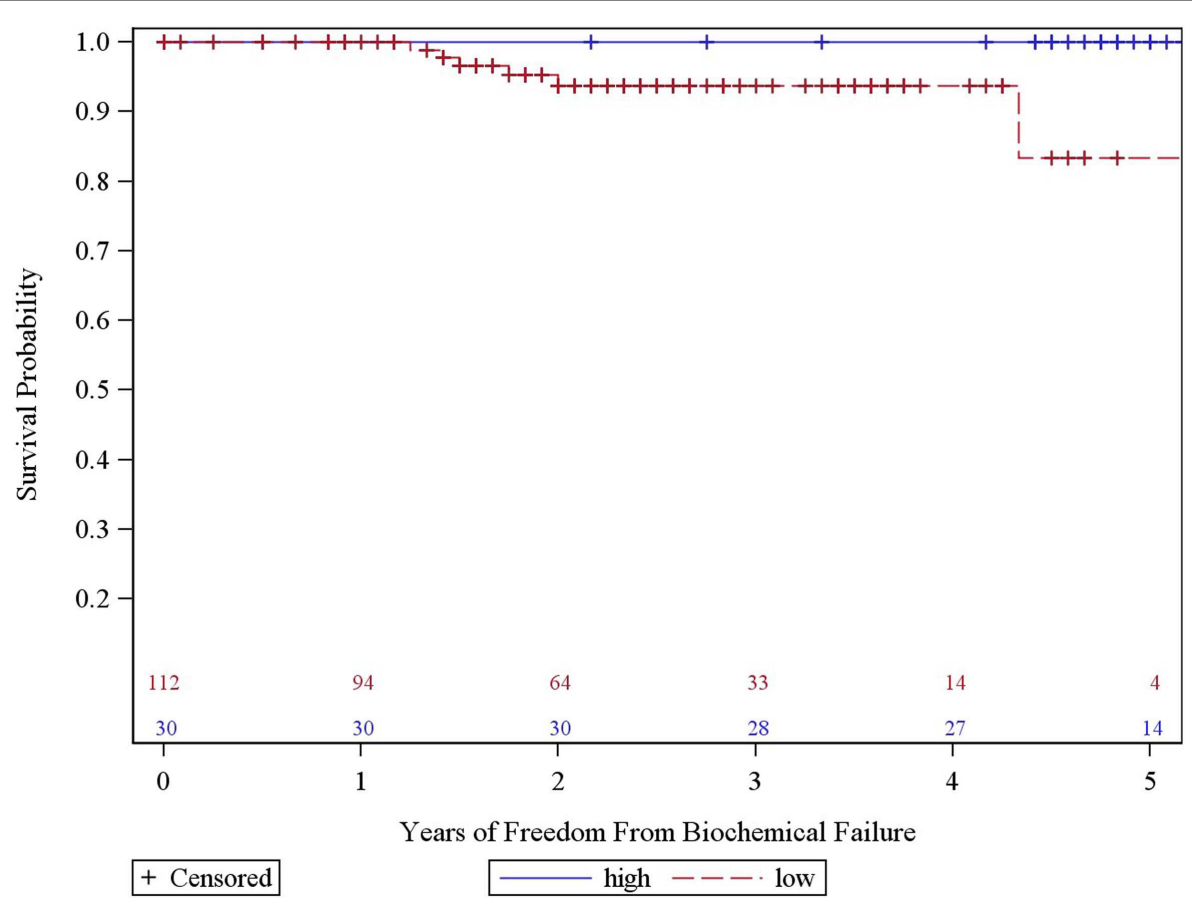

FIGURE 2 | Years of freedom from biochemical failure by dose for all patients. 
A statistically significant decrement in 5-year actuarial FFBF was noted with increasing GS from 95.45 to $93.4 \%$ for GS $5 / 6$ and 7 compared with $76.2 \%$ for $\mathrm{GS} \geq 8, p=0.03$, hazard ratio was 12.5 for GS $\geq 8$ vs. 3.5 for GS 7 vs. 1.0 for GS $\leq 6$ (Table 2, Figure 3 ). Pretreatment PSA, T-stage, age, use of ADT, and risk stratification groups were not significant with respect to 5-year FFBF.

Multivariate analysis evaluated the most significant factors in univariate analysis including risk stratification groups, GS, and dose. GS and dose were the most predictive factors in multivariate analysis but did not reach statistical significance at the $p<0.05$ level. Of note, with only six failures, we only have the power to detect a single significant predictor of failure in the multivariate analysis.

\section{TOXICITY}

Acute grade II GU toxicities were seen in $28 \%(n=32 / 113)$ and grade III GU toxicities were seen in $2 \%(n=3 / 142)$. Two of the three grade III acute GU toxicities manifested as frequency at least every hour and the other experienced gross hematuria. The patient who experienced gross hematuria had grade II baseline score with multiple urinary tract infections and transurethral resection of the prostate (TURP) prior to CyberKnife. All three patients' toxicities resolved within 3 months of treatment. However, one case of frequency at least every hour reappeared as a late toxicity 6 months after treatment. Acute gastrointestinal (GI) toxicity was limited to $4 \%(5 / 141)$ grade II toxicity with no acute grade III GI toxicity.

At most recent follow-up, persistent toxicities were limited to grade II GU of 14\% (18/132) and grade II GI of 3\% (4/141). For these persistent GI toxicities, there was no difference between dose groups; however, for persistent grade II GU toxicities, the low dose group observed less than the high dose group at 10 (10/102) and $27 \%(8 / 30)$, respectively. Of note there were three late grade III GU toxicities $2 \%(n=3 / 142)$, of which all three grade III patients were in the high dose group. One patient with a $162 \mathrm{~cm}^{3} \mathrm{CTV}$ (the largest prostate in our patient sample) had acute grade III frequency that resolved within 2 weeks of alpha-blocker and prophylactic antibiotic treatment, but recurred 6 months later. His symptoms improved to grade II immediately after TURP, which was preformed 13 months after SBRT. Pathology of the resected tissue was negative for tumor. The second patient with late grade III GU toxicity experienced urinary retention. He had benign prostate hypertrophy $(\mathrm{BPH})$ and grade II symptoms at baseline. At 14 months following SBRT, his symptoms progressed to grade III then completely subsided following laser TURP. All three grade III toxicities resolved at last follow to grade II or below.

Erectile dysfunction (ED) was assessed for the 48 patients who were potent before SBRT and did not receive ADT. At last followup, $13 \%$ lost the ability to achieve erections strong enough for penetration and required ED medication for intercourse. Six percent of patients who were potent before SBRT and did not receive ADT developed ED refractory to medical treatment.

\section{DISCUSSION}

In this updated study, we double the original number of patients and again report excellent FFBF rates with acceptable toxicity. The observed FFBF rates are concordant with other published SBRT outcomes for localized prostate cancer (Table 3) $(3-5,18)$.

To our knowledge, this is the first SBRT prostate cancer data base reporting outcome stratified retrospectively by the new version 2.2014 NCCN risk groups. In addition, we compare the

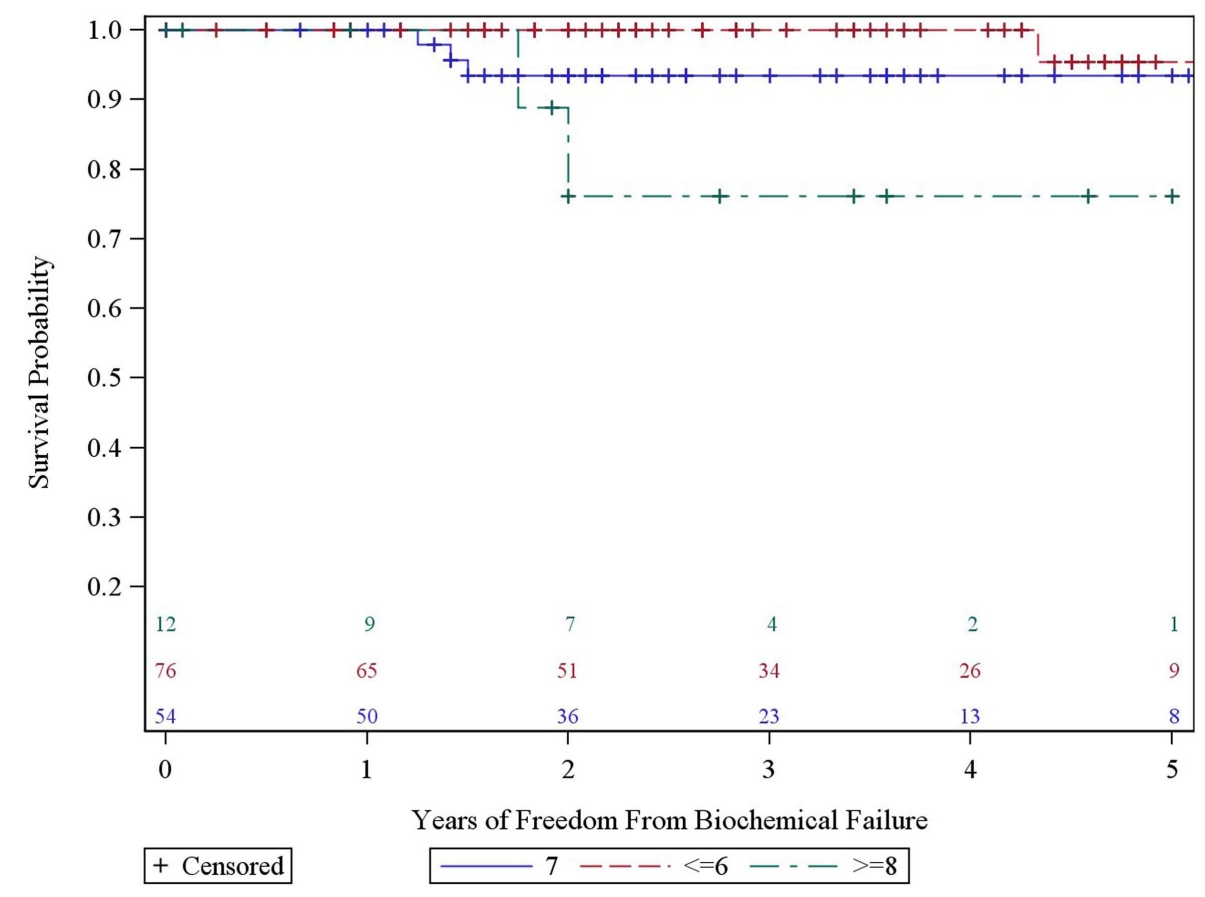

FIGURE 3 | Years of freedom from biochemical failure by Gleason score for all patients 
Table 3 | SBRT publications for treatment of prostate cancer with 5 year actuarial \%FFBF.

\begin{tabular}{|c|c|c|c|c|c|c|c|}
\hline \multirow[t]{2}{*}{ Study author } & \multirow[t]{2}{*}{ Patient population } & \multirow{2}{*}{$\begin{array}{l}\text { Number of } \\
\text { patients }\end{array}$} & \multirow[t]{2}{*}{ Dose } & \multirow{2}{*}{$\begin{array}{l}\text { Median follow-up } \\
\text { (months) }\end{array}$} & \multicolumn{3}{|c|}{ 5-year \%FFBF } \\
\hline & & & & & Low & Intermediate & High \\
\hline Freeman and King (5) & Low risk only, 2 institutions & 41 & $35 \mathrm{~Gy} / 5,36.25 \mathrm{~Gy} / 5$ & 60 & 93 & - & - \\
\hline Kang et al. (18) & 3 risk groups, single institution & 44 & $32 \mathrm{~Gy} / 4,34 \mathrm{~Gy} / 4,36 \mathrm{~Gy} / 4$ & 40 & 100 & 100 & 91 \\
\hline King et al. (6) & 3 risk groups, 8 institutions & 1100 & $36.25 G y / 4-5$ & 36 & 95 & 84 & 81 \\
\hline Katz et al. (19) & 3 risk groups, single institution & 304 & $35 \mathrm{~Gy} / 5,36.25 \mathrm{~Gy} / 5$ & 60 & 97 & 91 & 74 \\
\hline Current study & 3 risk groups, single institution & 142 & $\begin{array}{l}35 \mathrm{~Gy} / 5,36.25 \mathrm{~Gy} / 5 \\
37.5 \mathrm{~Gy} / 5\end{array}$ & 38 & 94 & $94^{*}$ & 84 \\
\hline
\end{tabular}

*Seven patients with T2c only included in intermediate group as per new NCCN guidelines.

prognostic value of other known factors in conjunction with NCCN risk groups in univariate and multivariate analysis. The current NCCN risk strategy separates out a very low risk group using many pathologic features in addition to the classic factors of low risk disease and separates out an unfavorable intermediate risk group to be combined with the high risk group. Our data suggest very little decrement in 5-year FFBF for very low, low, and favorable intermediate groups until at least two intermediate risk factors are present (unfavorable intermediate), which is grouped with high risk in the version 2.2014 NCCN guidelines.

Our data also suggest that GS may be the most important prognosticator being even more important than risk group stratification. The hazard for GS 8 is over 12 fold than that of GS 5-6 and 9 fold than that of GS 7 in our series with $p=0.03$. Katz et al. observed a decrease in 5 year FFBF for patients within the intermediate risk group with GS $4+3$ vs. all others from 95 to $84 \%$ (4). Katz et al. more recently reported their unfavorable intermediate and high risk groups utilizing the NCCN version 2.2014 criteria. Comparable to our series, only $47 \%$ of their high risk patients received androgen deprivation with no difference in FFBF noted in multivariate analysis. PSA was significant for outcome while GS was not which differs from our series (19).

In our previous experience with 3-year actuarial FFBF rates, we noted a statistically significant dose response for the intermediate and high risk patients (14). In the current study, which includes a larger sample size and longer follow-up, we continue to observe a significant increase in 5-year FFBR between high and low dose groups $(p=0.05)$. The sample size of the high dose group $(n=30)$ is about one-fourth of the size of the low dose group; nevertheless, no BF occurred in the high-dose group despite the longer median follow-up (57 vs. 33 months). This may come at the cost of increased persistent late GU toxicity with $27 \%$ grade II toxicities (compared to $10 \%$ in the low dose group). However, it is also important to note that no grade III GU toxicities persist at most recent follow-up.

A recently published multi-institutional consortium from eight independent phase 2 studies has pooled together 1100 patients with localized prostate cancer $(3,6)$. Patients had been treated definitively with CyberKnife SBRT, having a median follow-up of 36 months and median dose of $36.25 \mathrm{~Gy}$ in $4-5$ fractions. The risk stratification consisted of $58 \%$ low risk, $30 \%$ intermediate risk, and $11 \%$ high risk. They reported a 5-year FFBF 95, 84, and $81 \%$, respectively. Their overall 5-year FFBF rate was 93\%, with 49 total patients $(4.9 \%)$ experienced failure. This experience parallels our own, despite having a larger percentage of high and intermediate risk patients in the current series. There was no observed dose response among the range of doses used in the consortium of 35$40 \mathrm{~Gy}$. In addition to this analysis, they used a validated nomogram to compare outcomes of SBRT to radical prostatectomy, external beam radiotherapy, and permanent brachytherapy at 5 years. Although our current study is retrospective, it is encouraging to note these three conventional modalities have historically shown FFBF rates comparable to CyberKnife SBRT in our study as well as the aforementioned consortium of eight phase 2 studies.

Our late grade III urinary toxicity is similar to that reported in the literature with no grade III GI toxicity $(4,5,18-21)$. In our experience, dose may increase this risk since all three patients in our series with late grade III urinary toxicity were in the high dose group of $37.5 \mathrm{~Gy}$. This has been also reported by Katz et al. where all patients in his series with grade III toxicity were in the $36.25 \mathrm{~Gy}$ group compared with his 35 Gy group (4).

Radiation therapy oncology group 0938 is a randomized phase II trial assessing hypofractionated regimens of $36.25 \mathrm{~Gy}$ in five fractions of 7.25 Gy, and 51.6 Gy in 12 fractions of $4.3 \mathrm{~Gy}$ (22). The patients in this protocol are favorable risk, and hypofractionated schemes were delivered via linear accelerator, CyberKnife, or proton therapy. The RTOG protocol included a fractionation scheme $(36.25 \mathrm{~Gy}$ in five fractions at 7.25 Gy), which was prescribed for $75 \%$ of patients in our study. Although the patient population in this protocol are all "favorable risk," the primary endpoint is first-year health-related quality of life (HRQOL) measured by Bowel and Urinary domains of the Expanded Prostate Cancer Index Composite (EPIC) instrument; therefore, the results will be meaningful from a toxicity standpoint and applicable to less favorable risk patients (i.e., intermediate and high risk patients). Favorable HRQOL and EPIC scores may further validate the dose of $36.25 \mathrm{~Gy}$ in five fractions as the new standard. Fortunately, this protocol met accrual goals and closed February 2014.

In conclusion, SBRT could be considered an appropriate alternative to other standard radiation treatment modalities for early stage prostate cancer with excellent 5-year FFBF rates and low toxicity. Clinical expertise is required, but SBRT can be safely delivered 
in a community practice setting. The NCCN version 2.2014 further refines the risk strata, which may help guide patients treatment choices. GS may be the most important and simple prognosticator for risk assessment. Further study is warranted for unfavorable intermediate and high risk patients whose numbers are limited in most SBRT series. The role of dose escalation is unclear due to conflicting reports on the benefit on FFBF and risk of toxicity and also warrants additional study.

\section{REFERENCES}

1. American Medical Association. ASTRO Model Policies for Stereotactic Body Radiation Therapy (SBRT) for Prostate Cancer. (2013). Available from: https://www.astro.org/uploadedFiles/Main_Site/Practice_Management/ Reimbursement/2013HPcoding\%20guidelines_SBRT_Final.pdf

2. Mohler JL, Kantoff PW, Armstrong AJ, Plimack ER, Pow-Sang JM, Raben D, et al. Prostate cancer, version 2.2014, clinical practice guidelines in oncology. J Natl Compr Cancer Netw (2014) 12:686-718.

3. King CR, Freeman D, Kaplan I, Fuller D, Bolzicco G, Collins S, et al. Stereotactic body radiotherapy for localized prostate cancer: pooled analysis from a multiinstitutional consortium of prospective phase II trials. Radiother Oncol (2013) 109:217-21. doi:10.1016/j.radonc.2013.08.030

4. Katz AJ, Santoro M, Diblasio F, Ashley R. Stereotactic body radiotherapy for localized prostate cancer: disease control and quality of life at 6 years. Radiat Oncol (2013) 8:118. doi:10.1186/1748-717X-8-118

5. Freeman DE, King C. Stereotactic body radiotherapy for low-risk prostate cancer: 5 year outcomes. Radiat Oncol (2011) 6:3. doi:10.1186/1748-717X-6-3

6. King CR, Collins S, Fuller D, Wang PC, Kupelian P, Steinberg M, et al. Heathrelated quality of life after stereotactic body radiation therapy for localized prostate cancer: results from a multi-institutional consortium of prospective trials. Int J Radiat Oncol Biol Phys (2013) 87(5):939-45. doi:10.1016/j.ijrobp. 2013.08.019

7. D'Amico AV, Whittington R, Malkowicz SB, Cote K, Loffredo M, Schultz D, et al. Biochemical outcome after radical prostatectomy or external beam radiation therapy for patients with clinically localized prostate carcinoma in the prostate specific antigen era. Cancer (2002) 95:281-6. doi:10.1002/cncr.10657

8. D'Amico AV, Cote K, Loffredo M, Renshaw AA, Schultz D. Determinants of prostate cancer-specific survival after radiation therapy for patients with clinically localized prostate cancer. J Clin Oncol (2002) 20:4567-73. doi:10.1200/ JCO.2002.03.061

9. Epstein JI, Walsh PC, Carmichael M, Brendler CB. Pathologic and clinical findings to predict tumor extent of nonpalpable (stage T1c) prostate cancer. JAMA (1994) 271:368-74. doi:10.1001/jama.1994.03510290050036

10. Chun FK, Haese A, Ahyai SA, Walz J, Suardi N, Capitanio U, et al. Critical assessment of tools to predict clinically insignificant prostate cancer at radical prostatectomy in contemporary men. Cancer (2008) 113:701-9. doi:10.1002/cncr.23610

11. Bastian PJ, Carter B, Bjartell A, Seitz M, Stanislaus P, Montorsi F, et al. Insignificant prostate cancer and active surveillance from definition to clinical implications. Eur Urol (2009) 55:1321-30. doi:10.1016/j.eururo.2009.02.028

12. Zumsteg ZS, Spratt DE, Pei I, Zhang Z, Yamada Y, Kollmeier M, et al. A new risk classification system for therapeutic decision making with intermediaterisk prostate cancer patients undergoing dose-escalated external beam radiation therapy. Eur Urol (2013) 64:895-902. doi:10.1016/j.eururo.2013.03.033
13. Reese AC, Pierorazio PM, Han M, Partin AW. Contemporary evaluation of the national comprehensive cancer network prostate cancer risk classification system. Urology (2012) 80:1075-9. doi:10.1016/j.urology.2012.07.040

14. Oliai C, Lanciano R, Sprandio B, Yang J, Lamond J, Arrigo S, et al. Stereotactic body radiation therapy for the primary treatment of localized prostate cancer. J Radiat Oncol (2013) 2(1):63-70. doi:10.1007/s13566-012-0067-2

15. Cox JD, Stetz J, Pajak TF. Toxicity criteria of the radiation therapy oncology group (RTOG) and the European organization for research and treatment of cancer (EORTC). Int J Radiat Oncol Biol Phys (1995) 31:1341-6. doi:10.1016/0360-3016(95)00060-C

16. Kaplan EL, Meier P. Nonparametric estimation from incomplete observations. J Am Stat Assoc. (1958) 53(282):457-81. doi:10.1080/01621459.1958.10501452

17. Kleinbaum DG, Klein M. Survival Analysis. A Self-Learning Text. New York, NY: Springer (2005).

18. Kang JK, Cho CK, Choi CW, Yoo S, Kim MS, Yang K, et al. Image guided stereotactic body radiation therapy for localized prostate cancer. Tumori (2011) 97:43-8.

19. Katz AJ, Kang J. Stereotactic body radiotherapy with or without external beam radiation as treatment for organ confined high-risk prostate carcinoma: a six year study. Radiat Oncol (2014) 9:1. doi:10.1186/1748-717X-9-1

20. Madsen BL, Hsi RA, Pham HT, Fowler JF, Esagui L, Corman J. Stereotactic hypofractionated accurate radiotherapy of the prostate (SHARP), 33.5 Gy in five fractions for localized disease: first clinical trial results. Int J Radiat Oncol Biol Phys (2007) 67(4):1099-105. doi:10.1016/j.ijrobp.2006.10.050

21. Chen LN, Suy S, Uhm S, Oermann EK, Ju AW, Chen V, et al. Stereotactic body radiation therapy (SBRT) for clinically localized prostate cancer: the Georgetown university experience. Radiat Oncol (2013) 8:58. doi:10.1186/1748-717X-8-58

22. Radiation Therapy Oncology Group (RTOG) Protocol \#0938: A Randomized Phase II Trial of Hypofractionated Radiotherapy For Favorable Risk Prostate Cancer-RTOG CCOP Study, Closed. (2014). Available from: http://www.rtog.org/ClinicalTrials/ProtocolTable/StudyDetails.aspx?study=0938

Conflict of Interest Statement: The authors Dr. Rachelle Lanciano, Dr. John Lamond, Dr. Stephen Arrigo, and Dr. Luther W. Brady each own a small percentage of the single unit CyberKnife at the Philadelphia CyberKnife Center. The other coauthors declare that the research was conducted in the absence of any commercial or financial relationships that could be construed as a potential conflict of interest.

Received: 01 September 2014; accepted: 20 October 2014; published online: 11 November 2014.

Citation: Bernetich M, Oliai C, Lanciano R, Hanlon A, Lamond J, Arrigo S, Yang J, Good M, Feng J, Brown R, Garber B, Mooreville M and Brady LW (2014) SBRT for the primary treatment of localized prostate cancer: the effect of Gleason score, dose and heterogeneity of intermediate risk on outcome utilizing 2.2014 NCCN risk stratification guidelines. Front. Oncol. 4:312. doi: 10.3389/fonc.2014.00312

This article was submitted to Radiation Oncology, a section of the journal Frontiers in Oncology.

Copyright (C) 2014 Bernetich, Oliai, Lanciano, Hanlon, Lamond, Arrigo, Yang, Good, Feng, Brown, Garber, Mooreville and Brady. This is an open-access article distributed under the terms of the Creative Commons Attribution License (CC BY). The use, distribution or reproduction in other forums is permitted, provided the original author(s) or licensor are credited and that the original publication in this journal is cited, in accordance with accepted academic practice. No use, distribution or reproduction is permitted which does not comply with these terms. 